Manuelle Medizin 2018 · 56:1

https://doi.org/10.1007/s00337-018-0383-8

Online publiziert: 12. Februar 2018

C) Springer Medizin Verlag $\mathrm{GmbH}$, ein Teil von Springer Nature 2018

\title{
Online-Einreichung von Manuskripten für die Zeitschrift Manuelle Medizin
}

- Beschleunigt den Begutachtungsprozess:

Das Redaktionssystem ermöglicht die nahtlose Verbindung zwischen Einreichung, Begutachtung, eventueller Revision und schließlich Veröffentlichung.

- Ihre Daten sind sicher: Sie werden auf dem zentralen Datenserver gespeichert, der regelmäßig gesichert wird, sodass ein Datenverlust nicht vorkommt.

- Mehr Transparenz: Autoren können jederzeit online den aktuellen Stand der Begutachtung ihres eingereichten Artikels verfolgen.

Weitere Informationen zum Verfassen und Einreichen von Manuskripten finden Sie in der nebenstehenden Box.

Ihre Redaktion

Manuelle Medizin

\section{Korrespondenzadresse}

\section{Sabine Ehlenbeck}

Fachzeitschriften Medizin/Psychologie

Springer Medizin

Springer Verlag GmbH

Tiergartenstr. 17, 69121 Heidelberg,

Deutschland

sabine.ehlenbeck@springernature.com

\section{Manuskripte online einreichen}

Manuskript verfassen

- Bitte folgen Sie auf der Homepage www. ManuelleMedizin.springer.de dem Link "Hinweise für Autoren".

- Hier finden Sie die Autorenleitfäden und Musterbeiträge mit Hinweisen für die Manuskriptgestaltung.

Manuskript einreichen

- Bitte folgen Sie auf der Homepage www. ManuelleMedizin.springer.de dem Link "Online einreichen".

- Bei der ersten Nutzung registrieren Sie sich bitte unter „Register". Mit Ihrem „Author Login" haben Sie bei jeder weiteren Publikation direkten Zugang zum System.

- Nach der Anmeldung können Sie unter „Submit a manuscript" Ihren Beitrag einreichen.

Sollten Sie noch Fragen zur Manuskripteinreichung haben, wenden Sie sich bitte an unser Editorial Office:

MaMe_office@springernature.com www.ManuelleMedizin.springer.de

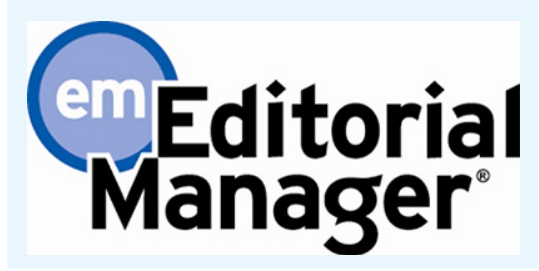

das überall an jedem Computer gleich aussieht. 